[When citing this chapter, refer to Behaviour 152 (2015) 493-520]

\title{
Bonobos and chimpanzees exploit helpful but not prohibitive gestures
}

\author{
Evan L. MacLean ${ }^{a, *}$ and Brian Hare ${ }^{a, b}$ \\ ${ }^{a}$ Department of Evolutionary Anthropology, Duke University, \\ Box 90383 Biological Sciences, Durham, NC 27705, USA \\ ${ }^{\mathrm{b}}$ Center for Cognitive Neuroscience, Duke University, Durham, NC 27705, USA \\ *Corresponding author's e-mail address: maclean@ duke.edu
}

Accepted 28 March 2014; published online 7 May 2014

\begin{abstract}
Previous research has shown that chimpanzees exploit the behavior of humans and conspecifics more readily in a competitive than a cooperative context. However, it is unknown whether bonobos, who outperform chimpanzees in some cooperative tasks, also show greater cognitive flexibility in competitive contexts. Here we tested the cooperative-competitive hypothesis further by comparing bonobos and chimpanzees in a series of tasks where a human gesture indicated the correct (cooperative) or incorrect (competitive) choice. A human either pointed cooperatively to the object a subject should choose, or competitively to the object subjects should avoid choosing. In contrast to previous research, subjects were most skilled at choosing the correct location when the communicator was cooperative and there were no major differences between bonobos and chimpanzees. Analysis of gaze direction revealed that in some cases subjects visually followed the direction of the experimenter's gesture despite choosing incorrectly, dissociating gesture following from gesture comprehension. This supports the hypothesis that, unlike human children, nonhuman apes respond to the direction of social gestures more readily than they understand the communicative intentions underlying them. We evaluate these findings in regard to previous studies comparing the cooperative and communicative skills of bonobos and chimpanzees.
\end{abstract}

\section{Keywords}

cognition, chimpanzee, bonobo, cooperation, competition.

\section{Introduction}

A range of animals are able to communicate in flexible ways depending on their audience, the context, and the state of the receiver (Searcy \& Nowicki, 2010). However, even the most adaptable animal communicators do not 
approach the flexibility observed in humans (Tomasello, 2008). Human infants are thought to begin developing skills for intentional communication as early as 9 months as evidenced first through their comprehension, and then production of pointing gestures (Carpenter et al., 1998; Behne et al., 2005). It has been proposed that the early expression of these pointing gestures is fundamental to the development of human cultural cognition and language (Carpenter et al., 1998; Butterworth, 2003) and the emergence of pointing in infants has become a central marker for the development of uniquely human social cognition (Tomasello, 2006).

The skills of other great apes have been examined to test whether the pointing abilities observed in human infants are unique to our species development (Hare, 2011). While nonhuman apes do regularly use manual gestures to flexibly communicate with one another in their natural interactions (Nishida, 1980; Goodall, 1986), evidence from observational and experimental studies suggest the same cognitive mechanisms are not always responsible for gestures in human and nonhuman apes (Call \& Tomasello, 2007). Longitudinal studies examining the natural gestures used by chimpanzees show that the production of these gestures develops relatively slowly through a process of social ritualization (Tomasello et al., 1994; Tomasello $\&$ Call, 1997). Nonhuman apes have largely only been observed to produce imperative gestures, and rarely if ever make declarative or informative gestures (Melis et al., 2006, 2009; Tomasello, 2006; Lyn et al., 2011). A series of studies also have suggested that in the most basic communicative contexts nonhuman apes show little flexibility in comprehending human gestures. Unlike human infants, mother-reared chimpanzees do not spontaneously use cooperative social gestures when searching for hidden objects or food (Call et al., 1998, 2000; Itakura et al., 1999; Barth et al., 2005). They do not show spontaneous skill even when the target of the gesture is made more obvious by placing the hiding locations as much as 2 m apart (Itakura et al., 1999; Herrmann \& Tomasello, 2006; but see Mulcahy \& Call, 2009). Moreover, once chimpanzees learn to use one human gesture to find food, they do not readily generalize this skill to a slightly different gesture. For example, when chimpanzees are trained to search in the location toward which a human extends their finger within a few centimeters, they no longer reliably follow the pointing gesture if the experimenter simply stands one meter away from the location to which he points (Povinelli et al., 1997, 1999). Finally, in a direct 
longitudinal comparison of human, bonobo and chimpanzee infants, only human infants showed spontaneous skill at reading human gestures (Wobber et al., 2013). Taken together, this work supports the hypothesis that the sudden and early emerging gestural flexibility seen in human infants is a completely derived trait in our lineage (Herrmann et al., 2007).

This conclusion has been challenged more recently. Some apes with extensive human socialization have shown skills similar to human infants in basic tests of their comprehension of human gestures (Call et al., 2000; Leavens et al., 2005; Lyn et al., 2010). Some researchers have suggested that wild as well as human socialized bonobos occasionally make declarative gestures (Veà \& Sabater-Pi, 1998; Lyn et al., 2011). Nonhuman great apes also adjust to their audience by making visual gestures more often when visible to the recipient, using tactile or vocal signals when not visible (Kaminski et al., 2004; Call \& Tomasello, 2007; Tempelmann et al., 2011), and signaling danger more often when the receiver is ignorant than knowledgeable of an imminent threat (Crockford et al., 2012). Finally, it has been suggested that apes only struggle to spontaneously read gestures in cooperative contexts but are skilled when interpreting the same gestures in a competitive context (Hare \& Tomasello, 2004). Almost universally nonhuman apes have been tested for their comprehension of human gestures in experimental contexts that require them to understand the cooperative-communicative intentions of a human experimenter. Yet primates rarely share information about the location of monopolizable food (Dittus, 1984; Coussi-Korbel, 1994; Hauser, 1996; Hirata \& Matsuzawa, 2001). This suggests that more flexible social cognitive skills will be observed in apes when they are competing (Hare, 2001). In support of this hypothesis chimpanzees were more skilled at locating hidden food using gestures when competing against the signaler than when cooperating with him (Hare \& Tomasello, 2004). Bonobos and chimpanzees were also more skilled at understanding the intentions behind a gesture meant to communicate which location they were prohibited from searching than when a similar gesture was made in a cooperative manner (Herrmann \& Tomasello, 2006). However, this study included only two bonobo subjects, limiting the ability for a direct comparison of the two Pan species.

These findings present the possibility that nonhuman apes can understand the communicative intentions behind a human gesture when searching for food in a competitive context; it is only that they do not understand the 
cooperative-communicative intentions of a human when the signaler is trying to help them locate monopolizable food. Moreover, it is not that apes fail to understand the meaning of human gestures because they cannot follow the directionality of these signals, because there is overwhelming evidence that apes follow the gaze direction of others to external targets (Tomasello et al., 1998, 1999, 2001, 2007b; MacLean \& Hare, 2012), but do not use these same cues to locate food in cooperative tasks (Call et al., 1998). This leads to the prediction that in cooperative contexts nonhuman apes may initially follow the direction of a human gesture, but do not reliably search in this location because they simply do not understand the informative motive behind the gesture in this context (Tomasello et al., 2007a). In the current series of studies we examine the ability of a group of bonobos and chimpanzees to use the pointing gestures of a human experimenter. We chose to investigate subjects' visual orienting and choice behavior in response to a pointing cue because many previous studies document that apes struggle to interpret this gesture in cooperative-communicative contexts (Call et al., 1998, 2000; Itakura et al., 1999; Barth et al., 2005). Thus, using a pointing gesture in both a cooperative and competitive context allows direct comparison to the previous literature, and also allows us to test the hypothesis that apes follow the direction of these cues faithfully, without understanding their communicative intent (Tomasello et al., 2007a).

Similar to Herrmann \& Tomasello (2006) we either cooperatively indicated where subjects should choose or competitively indicated where subjects were 'prohibited' from choosing. In order to test the orienting hypothesis we also varied whether the food being chosen was visible or hidden and we coded from video the first orienting response of subjects after the experimenter pointed. We predicted that subjects would choose correctly when the experimenter's gesture was prohibitive, but not cooperative, but would first orient toward the cued location in both contexts. This would support the idea that nonhuman apes follow the direction of pointing gestures, but do not understand the cooperative-communicative intentions underlying them. Lastly we directly compared the performance of bonobos and chimpanzees to assess whether bonobos, who outperform chimpanzees in some cooperative tasks (Hare et al., 2007; Tan \& Hare, 2013), and are affected by human competitors (Rosati \& Hare, 2012), also show greater proficiency in the cooperative-communicative object choice paradigm. 


\section{General methods}

All chimpanzee subjects live, and were tested at the Tchimpounga Sanctary, Pointe Noire, Republic of Congo. Bonobo subjects live and were tested at Lola ya Bonobo, Kinshasa, Democratic Republic of Congo. For a more detailed description of these field sites see Wobber \& Hare (2011). Subjects were tested in a familiar dormitory room with metal grid walls. Subject demographics and experiment participation are shown in Table 1 . The majority of subjects for both species (19 bonobos, 13 chimpanzees) had some previous experience in object choice tasks using cooperative-communicative gestures (see Table 1). All subjects were relatively unfamiliar with the experimenter (E1) prior to the experiment (E1 had briefly participated in research with some subjects approximately 1 year beforehand). Food was hidden under small plastic bowls $(17.5 \times 5.5 \mathrm{~cm})$ positioned on a table with a sliding platform $(50 \times 81 \mathrm{~cm})$ used to present subjects with the choices on each trial. On trials when food was hidden in containers a large plastic occluder was used to hide the baiting process from the subject. The sliding platform was marked in two places to delineate three $27 \mathrm{~cm}$ areas (left, center and right) used in scoring choices (see below). All trials were recorded from two video cameras. The first camera was positioned behind and to the side of E1 and recorded E1, the subject and procedure. The second camera was mounted to the rear of the table where choices were located and recorded the subject's face during trials (Figure 1). This angle was used to score looking behavior.

Data were analyzed using nonparametric statistics in IMB SPSS Version 20. We compared performance to chance expectation using one-sample Wilcoxon signed-rank tests. We compared performance between conditions and species using Mann-Whitney $U$-tests, and assessed learning between sessions using Related-Samples Wilcoxon signed-rank tests. We report the standardized test statistic ( $T$ ) from each of these tests throughout.

\section{Experiment 1}

In Experiment 1 subjects were introduced to the experimenter either as a cooperator, who shared food, or a competitor who stole food from the subject. Apes then participated in a series of object-choice trials in which the experimenter either helpfully pointed to the (visible) food item that subjects should choose (cooperative condition), or prohibitively pointed to the item that subjects should not choose (competitive condition). 
Apes exploit helpful but not prohibitive gestures

499 [253]

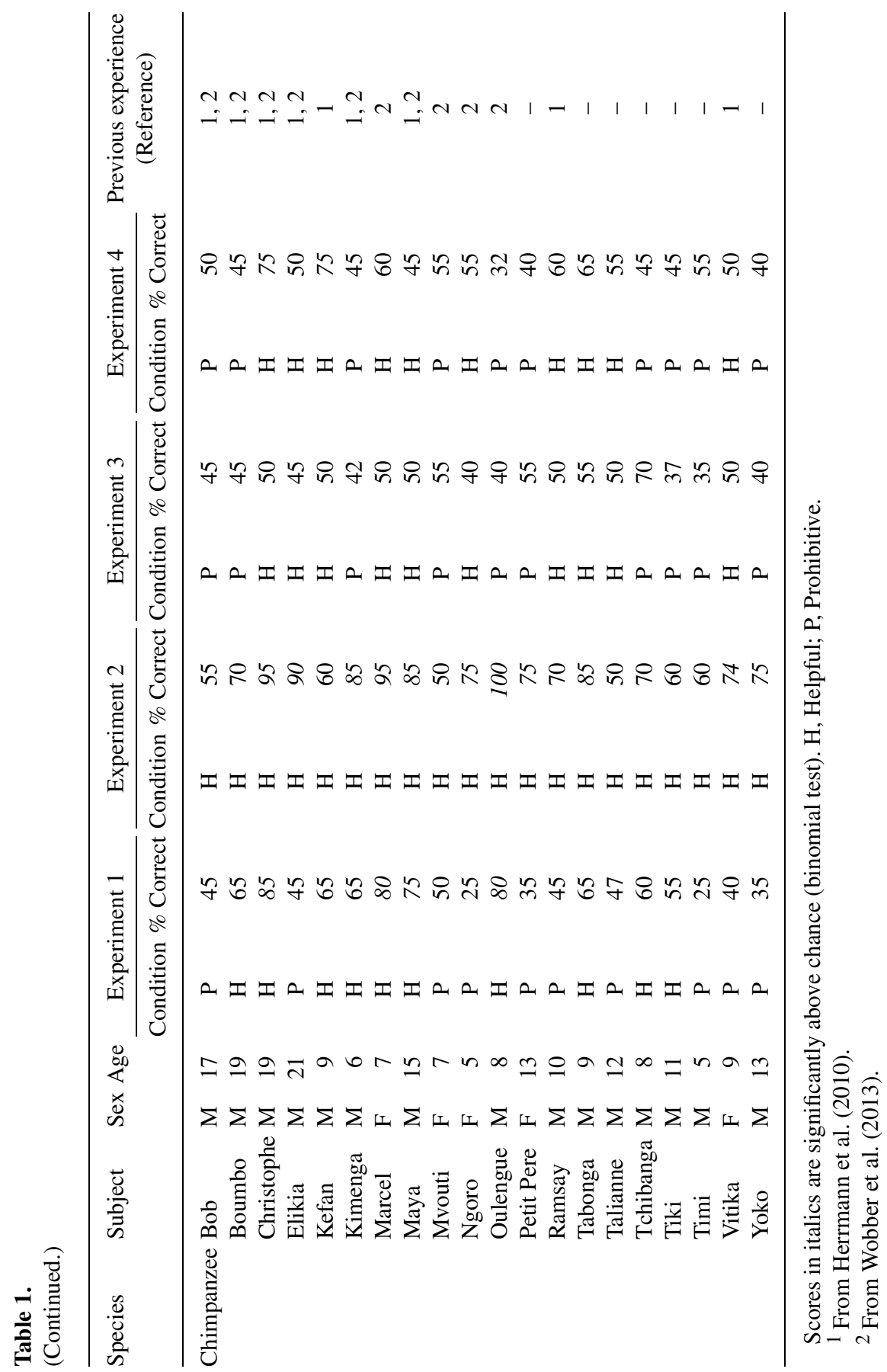



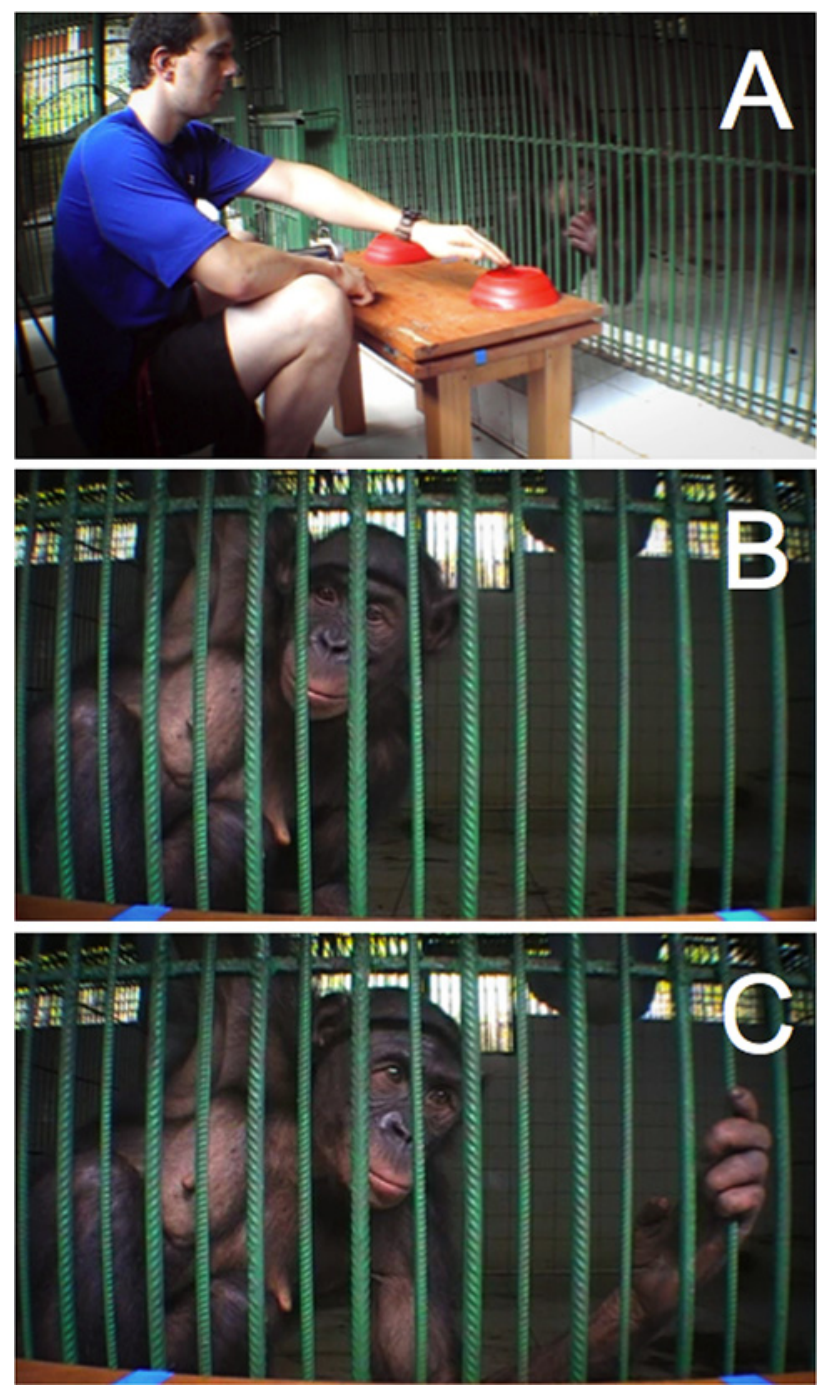

Figure 1. (A) The pointing gesture used in Experiments 1 and 2. Panels B and C show images from the coding camera corresponding to a subject looking at choices to the left (B) or right $(\mathrm{C})$ of the blue lines defining the choice areas. This figure is published in colour in the online edition of this journal, which can be accessed via http://booksandjournals.brillonline. com/content/journals/1568539x. 


\subsection{Method}

\subsubsection{Subjects}

We tested 20 chimpanzees (Table 1; 15 males, 5 females, mean age $=11$ years) and 20 bonobos (Table 1; 14 males, 6 females, mean age $=9$ years).

\subsubsection{Procedure}

3.1.2.1. Familiarization. We conducted 4 familiarization trials at the beginning of each session to introduce subjects to E1's cooperative or competitive motive. In each of these trials E1 placed a banana piece in the center of the table and slid the platform forward allowing subjects to reach for the food. Choices were defined as subjects touching or positioning their hand over the marked center area of the table where the food was positioned. In the cooperative condition E1 allowed subjects to retrieve the food. In the competitive condition E1 pulled the sliding platform away from subjects as they reached for the food, preventing them from obtaining it. We conducted these familiarization trials immediately before each of the two test sessions.

3.1.2.2. Test. In test trials E1 placed a piece of banana on each side of the table. He then called the subject's name and pointed to one of the banana pieces. The pointing gesture was performed with the contralateral arm, whole hand outstretched, fingers approximately $5 \mathrm{~cm}$ from the banana. E1 held this gesture for approx. $1 \mathrm{~s}$ before sliding the table forward for subjects to choose, maintaining the gesture until subjects made a choice or the trial timed out (see Design and analysis). Choices were defined as subjects touching or positioning their hand over the left or right marked areas of the table. Touching or positioning the hand over the center area was disregarded and E1 waited for subjects to choose either the left or right option. If a subject used both hands attempting to choose two options simultaneously, E1 retracted the table and repeated the trial. In the cooperative condition, if subjects chose the food E1 pointed to they were allowed to consume the reward, whereas choices to the non-indicated food ended the trial with no reward. In contrast, in the competitive condition if subjects chose the food E1 pointed to, E1 withdrew the table ending the trial, whereas subjects were allowed to consume the reward if they chose the non-indicated food. Therefore, in both conditions E1 provided subjects with the same social cue, but the meaning of this cue differed between the cooperative and competitive conditions. 


\subsubsection{Design and analysis. We conducted two 10-trial sessions with} each subject. These sessions were separated by a 5-min break. Half of the subjects within each species were tested in the cooperative condition while the other half were tested in the competitive condition. The location (left or right) that E1 pointed to was counterbalanced within each session. Subjects were allowed $30 \mathrm{~s}$ to choose on each trial and if no choice was made during this time the trial was scored as 'no choice' and the next trial was conducted. We used the percentage of correct choices (including only trials in which subjects made a choice) as the dependent measure for analysis of choice behavior. From video, we recorded the direction of subjects looking behavior (Figure 1) on each trial during the period beginning when E1 gave the cue, and ending when he pushed the platform forward for subjects to choose. Only the direction of the first look (left or right) was recorded and if subjects did not look to either location in this period the trial was scored as 'no look'. If the subject's face was out of view during the coding window, the trial was scored as 'off camera' and excluded from analysis. Because the lighting conditions and enclosure mesh varied between testing rooms, only a subset of videos could be coded for looking behavior (bonobo $N=11$, chimpanzee $N=8$ ). A second individual blind to the condition and hypotheses scored looking behavior and subjects' choices in $20 \%$ of trials to assess interobserver reliability. Inter-observer reliability was good for looking direction (kappa $=0.73$; Altman, 1991), very good for choice location (kappa $=0.93$ ), and scores from the primary coder were retained in cases of disagreement.

\subsection{Results}

\subsubsection{Choice accuracy}

Subjects in the cooperative condition chose the food that E1 pointed to significantly more often than expected by chance (mean $=69 \pm 3 \%$ correct, $T=3.73, N=20, p<0.01$ ) and showed no effect of learning between the first and second sessions $(T=-0.17, N=20, p=0.87)$. Eight subjects ( 4 bonobos, 4 chimpanzees) performed above chance expectation as individuals in the cooperative condition (binomial tests, $p<0.05$ ). Subjects in the competitive condition also tended to choose the food that E1 pointed to, leading to overall scores significantly worse than chance expectation (mean $=42 \pm 2 \%$ correct, $T=-2.99, N=20, p<0.01$ ). However, subjects in the competitive condition improved between the first and second test sessions, decreasing their tendency to choose the food that E1 pointed to 
$\square$ Cooperation $\square$ Competition

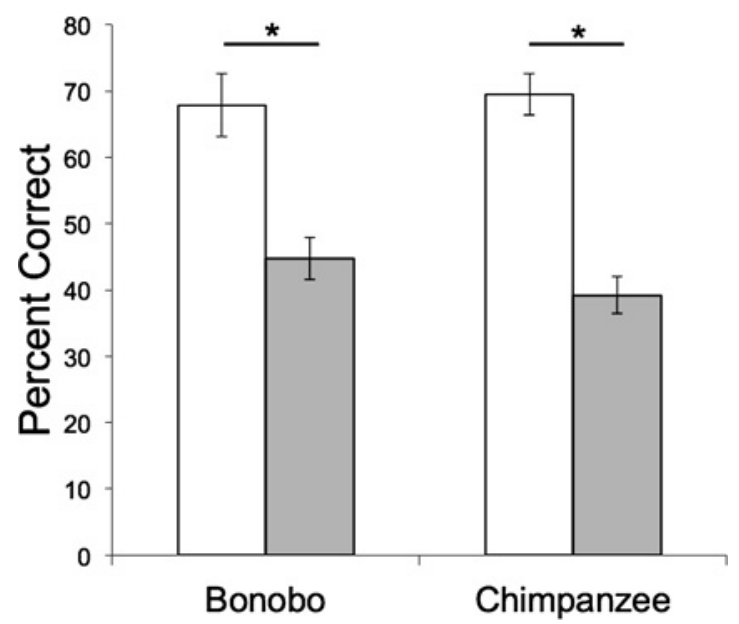

Figure 2. The results from Experiment 1. Both bonobos and chimpanzees performed better when E1's gesture cooperatively indicated the correct choice than when his gesture prohibitively indicated the incorrect choice.

over time $($ session 1 mean $=36 \pm 3 \%$ correct; session 2 mean $=48 \pm 3 \%$ correct; $T=2.54, N=20, p=0.01)$. At the individual level, no subjects performed above chance expectation in the competitive condition (binomial tests, $p>0.05$ ). Overall performance was significantly higher in the cooperative than the competitive condition in both species (Figure 2; chimpanzees: $T=3.80, N=20, p<0.01$; bonobos: $T=3.24, N=20, p<0.01$ ).

There were no species differences in overall performance in either the cooperative or competitive conditions (cooperation: $T=0.45, N=20$, $p=0.68$; competition: $T=-1.65, N=20, p=0.11)$. However, in the competitive condition chimpanzees improved between the two test sessions ( $T=2.46, N=10, p=0.01)$, but bonobos $\operatorname{did} \operatorname{not}(T=0.67, N=10$, $p=0.50)$.

\subsubsection{Looking measures}

For the subset of individuals for whom we were able to code looking behavior, we analyzed the pattern of looking behavior following E1's gesture, and the relationship between looking direction and subsequent choice accuracy. Subjects in the cooperation condition looked at one of the two items during the coding window in $95 \pm 2 \%$ of trials. Of these trials, their first look was to the item that E1 pointed to $91 \%$ of the time. Thus subjects visually followed 
E1's gesture to the correct item significantly more often than they chose this item $(T=2.52, N=8, p=0.01)$. After an initial look to the correct location these subjects chose with $73 \pm 5 \%$ accuracy, while choices following an initial look to the incorrect location tended to be less accurate $(50 \pm 22 \%$ correct; $T=-0.81, N=5, p=0.42$ ). Subjects in the competitive condition looked to one of the two items during the coding window in $88 \pm 3 \%$ of trials. Of these trials, their first look was to the item that E1 pointed to (the incorrect choice) $88 \%$ of the time. After initially looking at this item subjects chose at a very low accuracy (mean $=36 \pm 3 \%$ ), whereas choices following a look to the item E1 did not point to were significantly more accurate (mean $=76 \pm 11 \% ; T=-2.29, N=10, p=0.02)$.

\subsection{Discussion}

The main finding from Experiment 1 was that apes of both species performed significantly better when the experimenter's gesture was cooperative, and indicated the object they should choose, than when the gesture was competitive, and indicated the object they should not choose. This result contrasts with previous studies in which chimpanzees used prohibitive gestures at higher levels than cooperative-communicative gestures (Herrmann $\&$ Tomasello, 2006). However, several features of our design may explain this discrepancy. First, the prohibitive gesture in our study indicated the object that subjects should not choose, whereas the gesture used by Hermann $\&$ Tomasello (2006) directed the subjects' attention to the object they should choose (though it was intended to prohibit subjects from doing so). Thus it may be that the gesture directed subjects' attention to the indicated object, strongly biasing them to choose this object regardless of whether the experimenter's gesture was helpful or prohibitive. Our analysis of looking data supports this hypothesis as subjects in both the cooperative and competitive condition initially oriented toward (and tended to choose) the indicated object on the majority of trials. Second, in this task food was visible to subjects at both the correct and incorrect locations, and the experimenter's gesture served to indicate which item the subject should select rather than informing the (ignorant) subject of where a single piece of food was hidden. Consequently subjects were not reliant on the experimenter's gesture to infer the location of the reward, and instead tended to select the first visible piece of food that they oriented toward. In Experiment 2 we address the impact of 
whether the food is hidden or visible by testing subjects using the same gesture from Experiment 1 in a context where the food is hidden under one of two containers.

\section{Experiment 2}

In Experiment 2 we tested subjects in a traditional object choice task where food was hidden in one of two containers and the experimenter provided a helpful pointing gesture to indicate the food's location.

\subsection{Method}

\subsubsection{Subjects}

We tested the same subjects from Experiment 1 (approximately 1 week later).

\subsubsection{Procedure}

4.1.2.1. Familiarization. We conducted 4 familiarization trials at the beginning of each session to introduce subjects to finding food underneath the containers used in test trials. On each trial E1 visibly placed a piece of banana under one of the two containers positioned at the right and left sides of the table and slid the platform forward for the subject to make a choice. Choices were defined as subjects touching or positioning their hand over the marked areas at the left or right sides of the table. If a subject chose the baited container, E1 lifted this container allowing the subject to eat the reward. If a subject chose the un-baited container, E1 lifted this container to show the subject that it was empty, retracted the table and visibly removed the banana from underneath the correct container. Subjects were required to correctly choose the baited container on at least 3/4 familiarization trials to advance to the test. All subjects met this criterion.

In test trials E1 blocked the subject's view of the table with the plastic occluder and baited or sham baited each of the containers. E1 then removed the occluder, performed the pointing gesture (identical to Experiment 1) to indicate the baited container, and pushed the platform forward for subjects to make a choice. If a subject chose the baited container, E1 lifted this container allowing the subject to eat the reward. If a subject chose the unbaited container, E1 lifted this container to show the subject that it was empty, retracted the table and visibly removed the banana from underneath the baited container. 


\subsubsection{Design and analysis}

We conducted two 10-trial sessions with each subject. These sessions were separated by a 5-min break. The location that the experimenter pointed to was counterbalanced within each session. Subjects were allowed $30 \mathrm{~s}$ to choose on each trial and if no choice was made during this time the trial was scored as 'no choice' and the next trial was conducted. We used the percentage of correct choices (including only trials where subjects made a choice) as the dependent measure for analysis of choice behavior. From video, we recorded the direction of subjects looking behavior using the same procedures (and subjects) from Experiment 1 (bonobo $N=11$, chimpanzee $N=8$ ). A second individual blind to the condition and hypotheses scored looking behavior and subjects' choices for $20 \%$ of trials to assess inter-observer reliability. Inter-observer reliability was very good for both measures (looking: kappa $=0.85$; choice: kappa $=0.95$; Altman, 1991) and scores from the primary coder were retained in cases of disagreement.

\subsection{Results}

\subsubsection{Choice accuracy}

Overall subjects chose the container that E1 pointed to significantly more often than chance expectation (mean $=73 \pm 2 \%$ correct, $T=5.35, N=40$, $p<0.01$ ). Nineteen subjects ( 8 bonobos, 11 chimpanzees) performed above chance at the individual level (binomial tests, $p<0.05$ ). Of these subjects, 12 individuals had previously participated in the cooperative condition in Experiment 1, whereas 7 subjects had previously participated in the competitive condition. Averaging data across both test sessions, subjects who had previously participated in the cooperative condition in Experiment 1 did not differ from subjects who had previously participated in the competitive condition ( $T=1.89, N=40, p=0.06)$. However, these groups did differ significantly in the first test session, with subjects who had previously participated in the cooperative condition performing better than subjects who had previously participated in the competitive condition (Figure 3; previously cooperative: $79 \pm 4 \%$ correct; previously competitive: $65 \pm 4 \%$ correct; $T=2.23, N=40, p=0.03$ ). Nonetheless, both groups performed above chance in the first test session (previously cooperative: $T=3.55, N=20$, $p<0.01$; previously competitive: $T=2.66, N=20, p=0.01$ ). There was no significant difference between these groups in the second test session $(T=1.25, N=40, p=0.21)$. 


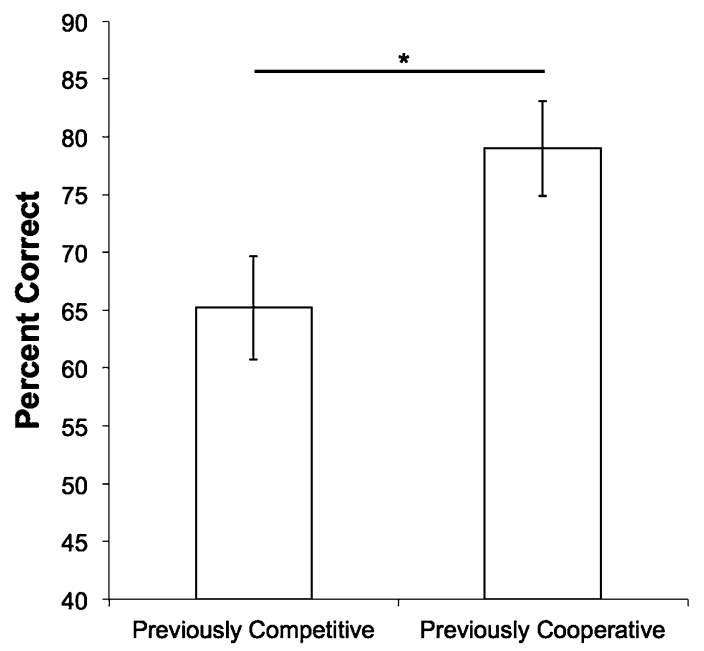

Figure 3. Performance in the first test session of Experiment 2 as a function of whether subjects previously participated in the cooperative or competitive condition in Experiment 1.

There were no differences between species regardless of whether subjects had previously participated in the cooperative $(T=0.77, N=20, p=0.48)$ or competitive ( $T=0.27, N=20, p=0.80$ ) condition in Experiment 1 . However, among the subjects that participated in the competitive condition of Experiment 1, chimpanzees performed above chance expectation in the first test session of this study (mean $=68 \pm 6 \%$ correct, $T=2.33, N=10$, $p=0.02$ ) whereas bonobos did not (mean $=62 \pm 7 \%$ correct, $T=1.36$, $N=10, p=0.17)$.

\subsubsection{Looking measures}

For the subset of individuals for whom we were able to code looking behavior, we analyzed the pattern of looking behavior following E1's gesture, and the relationship between looking direction and subsequent choice accuracy. Overall subjects looked toward one of the two containers during the coding window on $95 \pm 1 \%$ of trials. Of these trials, they first looked to the location that E1 pointed to $89 \%$ of the time. Following an initial look to the correct location subjects responded correctly on $78 \pm 3 \%$ of trials. In contrast, when subjects first looked toward the incorrect option the chose correctly only $48 \pm 10 \%$ of the time. The difference in accuracy following initial looks toward the correct and incorrect containers was significant ( $T=2.33, N=16, p=0.02$ ). Lastly, we compared whether subjects who 
participated in the cooperative condition of Experiment 1 were more likely to choose the location that they initially oriented toward more often when the food was visible (Experiment 1) than when the food was hidden (Experiment 2). Subjects chose the object they initially oriented toward at similar frequencies between these conditions (food visible: mean $=72 \pm 4 \%$; food hidden: mean $=70 \pm 6 \% ; T=-0.28, p=0.78$ ).

\subsection{Discussion}

Overall, subjects of both species used the cooperative-communicative gesture at high levels, and nearly half of our subjects performed above chance expectation at the individual level. These unusually high levels of performance may be due to the saliency of our pointing gesture, which was proximal to the indicated container (approx. $5 \mathrm{~cm}$ ), and was sustained until subjects had made a choice. Indeed, previous research indicates that cues conferring local enhancement generally facilitate performance in this type of task (Itakura et al., 1999; Miklósi \& Soproni, 2006; Schmitt et al., 2014). Moreover, the similarity in performance between Experiment 1 (cooperative condition) and Experiment 2 indicates that it was most likely the salience of the pointing cue, not simply that food was visible, that was responsible for subjects' high accuracy with the cooperative cue in Experiment 1.

Interestingly, in the first session of Experiment 2, subjects who had previously interacted with the experimenter as a competitor used the experimenters' cooperative gestures at lower levels than subjects who had previously interacted with the experimenter as a cooperator. Thus, even though this experiment was conducted approximately 1 week after Experiment 1, subjects' previous experience constrained or facilitated their initial ability to use cooperative-communicative cues in this study. However, after minimal experience interacting with the experimenter as a cooperator (10 trials), the difference between these groups abated. Thus, previous experience interacting with humans (both immediate and long term), is likely to influence apes' behavior in cooperative-communicative tasks (Lyn et al., 2010).

Finally, analysis of looking behavior in this study largely echoed the findings from Experiment 1 . When subjects initially looked toward the correct container, they chose with very high accuracy whereas choices following an initial look to the incorrect location tended to be inaccurate. However, on $\sim 20 \%$ of trials, subjects did initially look to the correct location, but subsequently chose incorrectly. Thus we found some support for the hypothesis that apes follow the direction of gestures in object choice tasks more 
readily than they understand the communicative intentions underlying them (Tomasello et al., 2007a). Our comparison of gaze direction following the gesture and the subject's subsequent choice in the hidden food and visible food manipulations of the first two experiments suggest that hiding the food has little effect on subject's performance. This argues against the previous suggestion that subjects perform poorly in the object choice task because they interpret the human's gesture as indicating the hiding location itself and not the food hidden within (Call et al., 1998).

\section{Experiment 3}

In Experiments 1 and 2, E1 gestured by extending his hand approx. $5 \mathrm{~cm}$ from the cued location. Opposite to our prediction that subjects would perform better when E1's gesture was prohibitive, both species performed best when E1 pointed cooperatively to the location they should choose, but poorly when E1 pointed to the location they were prohibited from choosing. However, the difference in performance between the cooperative and competitive conditions in Experiment 1 may simply reflect an effect of local enhancement in which the physical proximity of E1's hand to one of the choices biased subjects to attend to and select this option. Thus, even if apes possess an advantage with competitive communication, this effect may have been overshadowed in Experiment 1 by strong local enhancement cues. Therefore in Experiment 3 we introduced a more subtle gesture in which E1's arm was equidistant from the two options, reducing the potential confound of local enhancement. As in Experiment 1, this gesture was used either cooperatively to indicate the object that subjects should choose, or prohibitively to indicate the object that subjects should not choose.

\subsection{Method}

\subsubsection{Subjects}

The subjects were the same as Experiments 1 and 2, with the exception that only 12 bonobos ( 8 males, 4 females, mean age $=8.3$ years ) were tested in this study. Experiment 3 was conducted approximately 1 week after Experiment 2.

\subsubsection{Procedure}

We conducted 4 familiarization trials, identical to those from Experiment 1 , at the beginning of each session to introduce subjects to E1's cooperative 

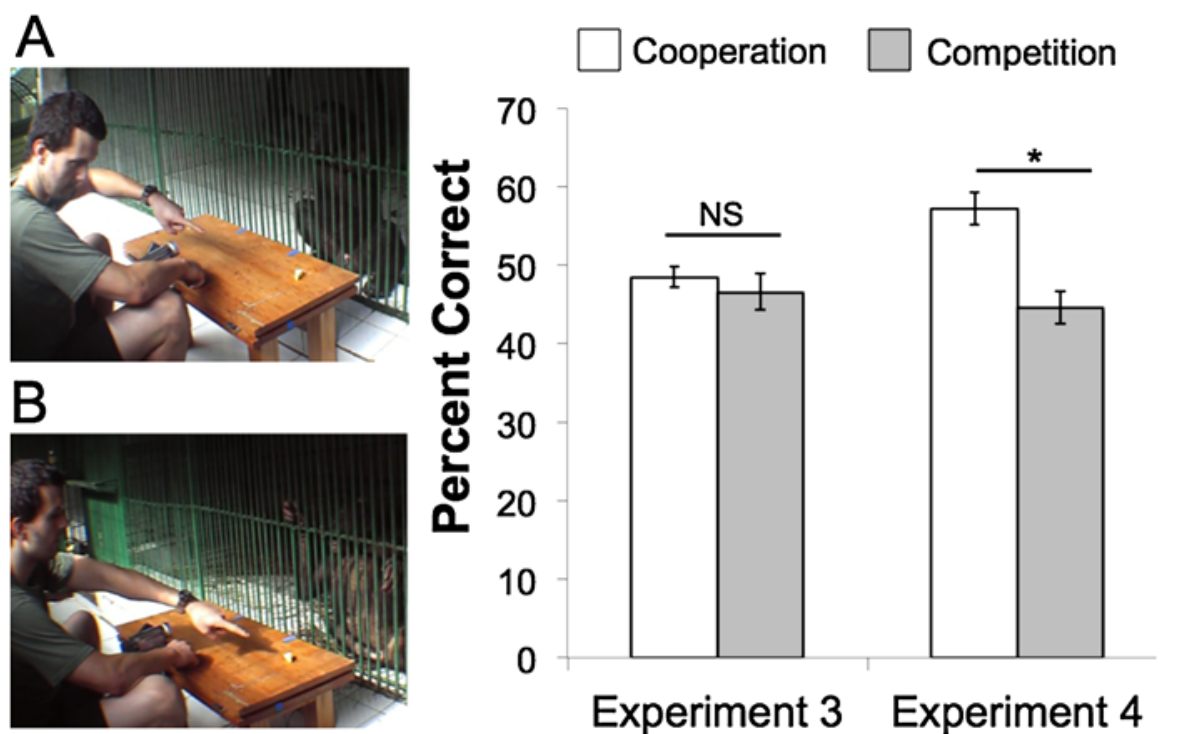

Experiment 3 Experiment 4

Figure 4. The pointing gesture and results from Experiments 3 and 4. In Experiment 3 subjects did not use a subtle pointing gesture (A) either when this gesture cooperatively indicated the correct location, or competitively indicated the incorrect location. In Experiment 4 E1 provided a more salient gesture (B) and subjects used this cue at higher levels in the cooperative than the competitive condition. This figure is published in colour in the online edition of this journal, which can be accessed via http://booksandjournals.brillonline. com/content/journals/1568539x.

or competitive motive. Test trials were identical to Experiment 1 with the exception that E1 provided subjects with a more subtle pointing gesture. Specifically, E1 held his hand centered in front of his chest, with his index finger extended toward one of the visible pieces of food (Figure 4A). While holding this gesture E1 said the subject's name and alternated his gaze between the subject and the location where he pointed two times. Following this cue E1 pushed the platform forward for subjects to choose. This cue was similar to that used by Hermann et al. (2007) with the exception that E1 stopped pointing before pushing the platform forward for subjects to choose. As in Experiment 1, half of the subjects within each species were tested in the cooperative condition, in which E1's gesture indicated the food that subjects should choose. For the other half of subjects (competitive condition), E1's gesture indicated the piece of food that subjects should not choose. 


\subsubsection{Design and analysis}

The design and analysis were identical to Experiment 1. As in Experiments 1-2 we attempted to score looking behavior between the time when E1 provided the cue and subjects' ultimate choice. However, the direction of subjects' looks was much less obvious in response to the subtle gesture used in this study, and inter-rater agreement (blind to the hypotheses and condition) was only moderate for looking direction (kappa: 0.56; Altman, 1991). Therefore we could not reliably code looking direction in this experiment and did not analyze these data. Inter-rater agreement for subjects' choices was very good (kappa: 0.96).

\subsection{Results}

Subjects in the cooperative condition did not choose the item that E1 pointed to more often than expected by chance (mean $=49 \pm 1 \%$ correct; $T=$ $-1.27, N=16, p=0.20$ ) and showed no learning between the first and second test session $(T=-1.71, N=16, p=0.09)$. Similarly, subjects in the competitive condition struggled to use E1's pointing gesture and also performed at chance levels (mean $=47 \pm 2 \%$ correct; $T=-1.58, N=16$, $p=0.11)$ with no learning between sessions $(T=-1.23, N=16, p=$ 0.22 ). No subjects performed above chance expectation at the individual level in either condition (binomial tests, $p>0.05$ ). A comparison of overall accuracy between the cooperative and competitive conditions revealed no significant difference (Figure 4; $T=1.06, N=32, p=0.31$ ).

There were no species differences in either the cooperative $(T=0.35$, $N=16, p=0.79)$ or competitive conditions $(T=-0.60, N=16, p=$ $0.56)$ and neither species performed above chance expectation in either the cooperative condition (chimpanzee: $T=-0.82, N=10, p=0.41$; bonobo: $T=-0.95, N=6, p=0.34$ ) or the competitive condition (chimpanzee: $T=-1.28, N=10, p=0.20$; bonobo: $T=-0.92, N=6, p=0.36$ ). Finally, performance did not differ between the cooperative and competitive conditions within either species (chimpanzee: $T=1.24, N=20, p=0.25$; bonobo: $T=0.08, N=12, p=1.0$ ).

\subsection{Discussion}

The main finding from Experiment 3 was that neither chimpanzees nor bonobos used a subtle pointing gesture (pointed finger centered in front of the experimenter's chest) either when this cue cooperatively indicated the correct choice, or prohibitively indicated the incorrect choice. While this cue 
eliminated the element of local enhancement that may have supported performance in Experiments 1 and 2, it also greatly reduced the salience of the cue leading to chance performance in both conditions. In contrast to Experiments 1 and 2, subjects' looking behavior could not be scored reliably, and it was not apparent that subjects visually followed the direction of the gestures.

\section{Experiment 4}

In Experiment 4 we tested apes in the same task from Experiment 3, but used a pointing gesture intermediate to that from Experiments 1 and 2, and Experiment 3.

\subsection{Method}

\subsubsection{Subjects}

We tested the same subjects from Experiment 3 (approx. 4 days later). Subjects were tested in the same condition (cooperative or competitive) that they had participated in during Experiment 3.

\subsubsection{Procedure}

The procedure was identical to Experiment 3 with the exception of the pointing gesture that $\mathrm{E} 1$ performed. In Experiment $4 \mathrm{E} 1$ pointed toward one of the food items with his index finger extended $\sim 25 \mathrm{~cm}$ from the item he pointed to (Figure 4B). This cue was maintained until subjects made a choice or the trial timed out. Thus the gesture used in this experiment was intermediate to that used in Experiment 1 (proximal pointing) and Experiment 3 (distal pointing).

\subsubsection{Design and analysis}

The design and analysis were identical to Experiment 3. Again we attempted to code the direction that subjects looked following E1's gesture. However, as in Experiment 3 the direction that subjects looked could not be judged reliably (kappa $=0.61$; Altman, 1991) and therefore was not included in analysis. Reliability for subjects' choices was very good (kappa $=0.93$ ). One bonobo subject in the competitive condition became reluctant to choose, making choices on only $4 / 10$ test trials in the first test session, and 0/10 trials in the second test session. Therefore we excluded this subject from analysis. 


\subsection{Results}

Overall, subjects in the cooperative condition chose the item that E1 pointed to significantly more often than expected by chance (mean $=57 \pm 2 \%$ correct; $T=2.88, N=16, p<0.01$ ) and showed no learning between the first and second test sessions $(T=-1.24, N=16, p=0.22)$. Two subjects (both chimpanzees) performed above chance at the individual level in the cooperative condition (binomial tests, $p<0.05$ ). Subjects in the competitive condition also tended to choose the item that $\mathrm{E} 1$ pointed to, leading to performance significantly worse than chance expectation (mean $=46 \pm 2 \%, T=-2.08$, $N=15, p=0.04)$. Subjects in the competitive condition showed no improvement between the first and second test sessions ( $T=0.45, N=15$, $p=0.66$ ), and no subject performed above chance expectation at the individual level (binomial tests, $p>0.05$ ). Overall subjects in the cooperative condition performed significantly better than subjects in the competitive condition (Figure 4; $T=3.55, N=31, p<0.01$ ).

There were no species differences in either the cooperative $(T=0.77$, $N=16, p=0.49)$ or the competitive condition $(T=-0.69, N=15$, $p=0.51)$. Consistent with the results across species, both chimpanzees and bonobos performed above chance in the cooperative condition (chimpanzees: $T=2.26, N=10, p=0.02$; bonobos: $T=2.03, N=6, p=0.04$ ), and tended to score below chance in the competitive condition (chimpanzees: $T=-1.90, N=10, p=0.06$; bonobos: $T=-0.92, N=5, p=0.36$ ). Similarly, performance was better in the cooperative than the competitive condition within both species (chimpanzee: $T=2.91, N=20, p<0.01$; bonobos: $T=2.02, N=11, p=0.05$ ).

Lastly, for subjects that participated in the same condition (cooperative or competitive) across Experiments 1, 3 and 4, we conducted a Friedman's analysis of variance by ranks to determine whether performance differed significantly across the three types of pointing gesture. For subjects in the cooperative condition performance varied significantly across cue types $\left(\chi^{2}=9.94\right.$, $N=9$, df $=2, p<0.01)$ and post-hoc tests revealed that performance in Experiment 1 was better than performance in Experiment 3, but that no other pairs differed. For subjects in the competitive condition performance also varied significantly across cue types $\left(\chi^{2}=9.33, N=8, \mathrm{df}=2, p=0.01\right)$, and post hoc tests revealed that performance was significantly worse in Experiment 1 than in Experiment 4, but that no other pairs differed. 


\subsection{Discussion}

The results from Experiment 4 are consistent with those from Experiment 1. Subjects of both species used the cooperative cue more readily than the competitive cue to guide their choice behavior. The main difference from Experiment 1 was that here, subjects were less skilled with the pointing gesture, most likely because E1's finger was less proximal to the indicated object. As in Experiment 3, subjects' initial orienting responses were not easily detected, and could not be scored reliably.

\section{General discussion}

Our findings provide no support for the competitive communicator hypothesis. In the three studies where subjects were tested in either a cooperative or competitive scenario, subjects in the competitive condition never responded above chance expectation, and were significantly worse than chance expectation in several cases. In contrast, many subjects in the cooperative condition responded above chance expectation in multiple contexts.

Interestingly we found some support for the hypothesis that apes respond to the direction of social gestures more readily than they understand the communicative intentions underlying them. In Experiments 1-2, subjects tended to follow E1's gesture to the correct referent, but only subsequently chose this object approx. $75 \%$ of the time. Thus apes followed the direction of the gesture considerably more often than they used the gesture to guide their choice. This tendency to look toward the correct choice more often than choosing it occurred both when the food was visible, or hidden from the subjects during the procedure. This argues against the possibility that apes struggle with the object choice task because they interpret the gesture as referring to the hiding container itself (which has no value to the subject), rather than the hidden food within it (Call et al., 1998, 2000). However, in Experiments 3 and 4 subjects' looking direction was less salient and could not be coded reliably. Given that looking direction was less clear in these contexts, it is possible that apes struggled to use the pointing gesture in part due to a failure to link the gesture to its referent. Therefore, determining whether apes follow the directionality of more subtle pointing gestures (such as those from Experiments 3 and 4) remains an important question for future research, and will likely be aided by improved technology for noninvasively 
monitoring eye movements (e.g., Hattori et al., 2010; Kano \& Tomonaga, 2011).

Our results raise the question of why we did not replicate the findings of previous research on this topic (e.g., Hare \& Tomasello, 2004; Herrmann \& Tomasello, 2006). One possible explanation could be subtle methodological differences between the different experiments. For example, in Hare \& Tomasello (2004) procedures were designed to maximize the competitive nature of the interaction. The human experimenter indicated displeasure when a subject chose the correct hiding location and when the subject chose incorrectly, the experimenter ate the food reward to clearly indicate that he was actively competing. It may be that these types of social cues are necessary to indicate that a human - who typically is neutral or helpful - is actively competing. Like previous studies (Hare \& Tomasello, 2004; Herrmann \& Tomasello, 2006), our experiments did not use behavioral criteria to verify that a cooperative or competitive relationship had been established prior to the test. Thus an important design consideration for future studies will be to determine the specific procedures required to effectively establish a cooperative or competitive relationship, as well as behavioral measures to assess the impact of these manipulations.

A second plausible explanation is that the competitive gestures used by Hare \& Tomasello (2004) and Herrmann \& Tomasello (2006), still directed subjects' attention to the correct response (e.g., a demonstrator reached toward the object unsuccessfully, or forbid subjects from selecting the object), whereas our competitive gesture directed subjects' attention to the object they should not choose. Consequently, in our studies subjects may have struggled to avoid inhibiting the incorrect response after the gesture directed their attention to this object. Indeed, in Experiments 1 and 2 subjects did visually orient toward the indicated location on the majority of trials, regardless of whether the experimenter's gesture was cooperative or competitive in nature. Therefore, the salience of this gesture may have inadvertently introduced a problem of inhibitory control in which subjects in the competitive condition were required to choose the location opposite to where their attention was originally directed. Given that inhibitory control actively constrains primate problem solving in many contexts (Santos et al., 1999; Amici et al., 2008; Vlamings et al., 2009; MacLean et al., in press), it may also partially explain the generally poor performance in the competitive conditions of these tasks. Therefore, it may be that the competitive advantage is only de- 
tectable when both the cooperative and competitive gestures initially direct attention toward the correct response, and there are few constraints imposed by inhibitory control.

Another important difference between our study and previous publications testing the competitive communicator hypothesis is differences in the rearing and experimental histories of the subjects. It is possible that the difference in results are in part a result of the daily human contact that the majority of our subjects receive from human surrogate mothers for the first several years they live at the sanctuary; they receive helpful pointing gestures from humans in a way that mother-reared zoo-born apes typically do not. Additionally, the majority of our subjects had some previous experience in cooperativecommunicative tasks (Table 1) perhaps facilitating their performance in these studies. As documented in other populations of apes, rearing history and previous experience can influence performance in these types of tasks in some cases (Tomasello \& Call, 2004; Lyn et al., 2010). Thus an important question for future research will be whether these findings generalize to novel gestures that subjects have no experience with prior to the test (e.g., foot pointing).

Regardless of which of these explanations best accounts for our data, it is clear that the competitive communication hypothesis has little power to explain the results of these studies. Therefore future research on the methodological variables that elicit a competitive response, and the role of other task demands (e.g., inhibiting a response when attention is directed toward the incorrect option) will be important for further evaluation of the competitive communicator hypothesis.

Lastly, we found no differences between bonobos and chimpanzees in either the cooperative or competitive conditions. Bonobos, who are characterized by higher social tolerance than chimpanzees (Hare et al., 2012), have been shown to outperform chimpanzees in some cooperative problemsolving tasks (Hare et al., 2007; Tan \& Hare, 2013; but see MacLean \& Hare, 2013). In addition, bonobos are sensitive to human competitors as their preferences for risk in a foraging task shifted in similar ways to chimpanzees when both species competed against a human (Rosati \& Hare, 2013). However, the explanation for the species differences in cooperative abilities implicates differences in social tolerance, not differences in an understanding of cooperative or communicative intentions (Hare et al., 2007). Our results lend further support for this interpretation, as the two Pan species appear not to differ in their understanding of cooperative or competitive gestures. 


\section{Acknowledgements}

We thank R. Atencia, L. Pharoah, D. Cox, K. Brown and the chimpanzee caretakers for facilitating research at Tchimpounga Chimpanzee Sanctuary, C. Andre, V. Dhanani, D. Morel, P. Mbonzo, F. Mehl and the bonobo caretakers for their assitance at Lola ya Bonobo, C. Boukindi, G. Shorland and $\mathrm{S}$. Kwetuenda for their assistance with data collection, and K. Leimberger, S. Laderman, and K. Almon for data coding. We also thank the Ministry of Scientific Research and Technological Inovation, Republic of Congo, and the Ministry of Scientific Research and Technology, Democratic Republic of Congo (permit: MIN.RS/SG/004/2009), for the permits under which this research was conducted. We thank the North Carolina Zoo, and chimpanzee zookeepers for supporting pilot studies of these experiments. We thank all members of the Hare group and Department of Evolutionary Anthropology at Duke University for their helpful comments on these studies. This work was supported in part by European Research Commission Advanced Grant Agreement 233297, National Institute of Health Grant 5 R03 HD070649-02, National Science Foundation grants NSF-BCS-08-27552 and NSF-BCS-1025172 to B.H.

\section{References}

Altman, D.G. (1991). Practical statistics for medical research. - Chapman and Hall, London.

Amici, F., Aureli, F. \& Call, J. (2008). Fission-fusion dynamics, behavioral flexibility, and inhibitory control in primates. - Curr. Biol. 18: 1415-1419.

Barth, J., Reaux, J.E. \& Povinelli, D.J. (2005). Chimpanzees' (Pan troglodytes) use of gaze cues in object-choice tasks: different methods yield different results. - Anim. Cogn. 8: 84-92.

Behne, T., Carpenter, M. \& Tomasello, M. (2005). One-year-olds comprehend the communicative intentions behind gestures in a hiding game. - Dev. Sci. 8: 492-499.

Butterworth, G. (2003). Pointing is the royal road to language for babies. - In: Pointing: where language, culture, and cognition meet (Kita, S., ed.). Lawrence Erlbaum Associates, Mahwah, NJ, p. 9-33.

Call, J. \& Tomasello, M. (2007). The gestural communication of apes and monkeys. Lawrence Erlbaum Associates, Publishers, Mahwah, N.J.

Call, J., Hare, B. \& Tomasello, M. (1998). Chimpanzee gaze following in an object-choice task. - Anim. Cogn. 1: 89-99.

Call, J., Agnetta, B. \& Tomasello, M. (2000). Cues that chimpanzees do and do not use to find hidden objects. - Anim. Cogn. 3: 23-24. 
Carpenter, M., Nagell, K. \& Tomasello, M. (1998). Social cognition, joint attention, and communicative competence from 9 to 15 months of age. - Monogr. Soc. Res. Child Dev. 63: $1-143$.

Coussi-Korbel, S. (1994). Learning to outwit a competitor in mangabeys (Cercocebus torquatus torquatus). - J. Comp. Psychol. 108: 164.

Crockford, C., Wittig, R.M., Mundry, R. \& Zuberbühler, K. (2012). Wild chimpanzees inform ignorant group members of danger. - Curr. Biol. 22: 142-146.

Dittus, W.P. (1984). Toque macaque food calls: semantic communication concerning food distribution in the environment. — Anim. Behav. 32: 470-477.

Goodall, J. (1986). The chimpanzees of gombe: patterns of behavior. - Belknap Press of Harvard University Press, Cambridge, MA.

Hare, B. (2001). Can competitive paradigms increase the validity of experiments on primate social cognition? - Anim. Cogn. 4: 269-280.

Hare, B. (2011). From hominoid to hominid mind: what changed and why? - Ann. Rev. Anthropol. 40: 293-309.

Hare, B. \& Tomasello, M. (2004). Chimpanzees are more skilful in competitive than in cooperative cognitive tasks. - Anim. Behav. 68: 571-581.

Hare, B., Melis, A.P., Woods, V., Hastings, S. \& Wrangham, R. (2007). Tolerance allows bonobos to outperform chimpanzees on a cooperative task. — Curr. Biol. 17: 619-623.

Hattori, Y., Kano, F. \& Tomonaga, M. (2010). Differential sensitivity to conspecific and allospecific cues in chimpanzees and humans: a comparative eye-tracking study. - Biol. Lett. 6: 610-613.

Hauser, M.D. (1996). The evolution of communication. - The MIT Press.

Herrmann, E. \& Tomasello, M. (2006). Apes' and children's understanding of cooperative and competitive motives in a communicative situation. — Dev. Sci. 9: 518-529.

Herrmann, E., Call, J., Hernandez-Lloreda, M.V., Hare, B. \& Tomasello, M. (2007). Humans have evolved specialized skills of social cognition: the cultural intelligence hypothesis. Science 317: 1360-1366.

Hirata, S. \& Matsuzawa, T. (2001). Tactics to obtain a hidden food item in chimpanzee pairs (Pan troglodytes). - Anim. Cogn. 4: 285-295.

Itakura, S., Agnetta, B., Hare, B. \& Tomasello, M. (1999). Chimpanzee use of human and conspecific social cues to locate hidden food. — Dev. Sci. 2: 448.

Kaminski, J., Call, J. \& Tomasello, M. (2004). Body orientation and face orientation: two factors controlling apes' begging behavior from humans. - Anim. Cogn. 7: 216-223.

Kano, F. \& Tomonaga, M. (2011). Species difference in the timing of gaze movement between chimpanzees and humans. - Anim. Cogn. 14: 879-892.

Leavens, D.A., Hopkins, W.D. \& Bard, K.A. (2005). Understanding the point of chimpanzee pointing - epigenesis and ecological validity. — Curr. Dir. Psychol. Sci. 14: 185-189.

Lyn, H., Greenfield, P.M., Savage-Rumbaugh, S., Gillespie-Lynch, K. \& Hopkins, W.D. (2011). Nonhuman primates do declare! A comparison of declarative symbol and gesture use in two children, two bonobos, and a chimpanzee. — Lang. Commun. 31: 63-74.

Lyn, H., Russell, J.L. \& Hopkins, W.D. (2010). The impact of environment on the comprehension of declarative communication in apes. - Psychol. Sci. 21: 360-365. 
MacLean, E.L. \& Hare, B. (2012). Bonobos and chimpanzees infer the target of another's attention. - Anim. Behav. 83: 345-353.

MacLean, E. \& Hare, B. (2013). Spontaneous triadic engagement in bonobos (Pan paniscus) and chimpanzees (Pan troglodytes). - J. Comp. Psychol. 127: 245-255.

MacLean, E.L., Hare, B., Nunn, C.L., Addessi, E., Amici, F., Anderson, R.C., et al. (in press). The evolution of self-control. - Proc. Natl. Acad. Sci. USA 111.

Melis, A.P., Hare, B. \& Tomasello, M. (2006). Chimpanzees recruit the best collaborators. Science 311: 1297-1300.

Melis, A.P., Hare, B. \& Tomasello, M. (2009). Chimpanzees coordinate in a negotiation game. — Evol. Hum. Behav. 30: 381-392.

Miklósi, A. \& Soproni, K. (2006). A comparative analysis of animals' understanding of the human pointing gesture. - Anim. Cogn. 9: 81-93.

Mulcahy, N.J. \& Call, J. (2009). The performance of bonobos (Pan paniscus), chimpanzees (Pan troglodytes), and orangutans (Pongo pygmaeus) in two versions of an object-choice task. — J. Comp. Psychol. 123: 304-309.

Nishida, T. (1980). The leaf-clipping display: a newly-discovered expressive gesture in wild chimpanzees. - J. Hum. Evol. 9: 117-128.

Povinelli, D.J., Bierschwale, D.T. \& Cech, C.G. (1999). Comprehension of seeing as a referential act in young children, but not juvenile chimpanzees. - Br. J. Dev. Psychol. 17: 37-60.

Povinelli, D.J., Reaux, J.E., Bierschwale, D.T., Allain, A.D. \& Simon, B.B. (1997). Exploitation of pointing as a referential gesture in young children, but not adolescent chimpanzees. — Cogn. Dev. 12: 327-365.

Rosati, A.G. \& Hare, B. (2012). Decision making across social contexts: competition increases preferences for risk in chimpanzees and bonobos. - Anim. Behav. 84: 869-879.

Santos, L.R., Ericson, B.N. \& Hauser, M.D. (1999). Constraints on problem solving and inhibition: object retrieval in cotton-top tamarins (Saguinus oedipus oedipus). — J. Comp. Psychol. 113: 186-193.

Schmitt, V., Schloegl, C. \& Fischer, J. (2014). Seeing the experimenter influences the response to pointing cues in long-tailed macaques. — Plos One 9: e91348.

Searcy, W.A. \& Nowicki, S. (2010). The evolution of animal communication: reliability and deception in signaling systems. - Princeton University Press, Princeton, NJ.

Tan, J. \& Hare, B. (2013). Bonobos share with strangers. — Plos One 8: e51922.

Tempelmann, S., Kaminski, J. \& Liebal, K. (2011). Focus on the essential: all great apes know when others are being attentive. - Anim. Cogn. 14: 433-439.

Tomasello, M. (2006). Why don't apes point? - In: Roots of human sociality: culture, cognition and interaction (Enfield, N.J. \& Levinson, S.C., eds). Berg, New York, NY, p. 506-524.

Tomasello, M. (2008). Origins of human communication. — MIT Press, Cambridge, MA.

Tomasello, M. \& Call, J. (1997). Primate cognition. — Oxford University Press, New York, NY.

Tomasello, M. \& Call, J. (2004). The role of humans in the cognitive development of apes revisited. - Anim. Cogn. 7: 213-215. 
Tomasello, M., Call, J., Nagell, K., Olguin, R. \& Carpenter, M. (1994). The learning and use of gestural signals by young chimpanzees: a trans-generational study. — Primates 35 : 137-154.

Tomasello, M., Call, J. \& Hare, B. (1998). Five primate species follow the visual gaze of conspecifics. - Anim. Behav. 55: 1063-1069.

Tomasello, M., Hare, B. \& Agnetta, B. (1999). Chimpanzees, Pan troglodytes, follow gaze direction geometrically. - Anim. Behav. 58: 769-777.

Tomasello, M., Hare, B. \& Fogleman, T. (2001). The ontogeny of gaze following in chimpanzees, Pan troglodytes, and Rhesus macaques, Macaca mulatta. - Anim. Behav. 61: 335-343.

Tomasello, M., Carpenter, M. \& Liszkowski, U. (2007a). A new look at infant pointing. Child Dev. 78: 705-722.

Tomasello, M., Hare, B., Lehmann, H. \& Call, J. (2007b). Reliance on head versus eyes in the gaze following of great apes and human infants: the cooperative eye hypothesis. J. Hum. Evol. 52: 314-320.

Veà, J. \& Sabater-Pi, J. (1998). Spontaneous pointing behaviour in the wild pygmy chimpanzee (Pan paniscus). — Folia Primatol. 69: 289-290.

Vlamings, P.H., Hare, B. \& Call, J. (2009). Reaching around barriers: the performance of the great apes and 3-5-year-old children. - Anim. Cogn. 13: 273-285.

Wobber, V., Herrmann, E., Hare, B., Wrangham, R. \& Tomasello, M. (2013). Differences in the early cognitive development of children and great apes. - Dev. Psychobiol. 56: 547-573.

Wobber, V.T. \& Hare, B. (2011). Psychological health of orphan bonobos and chimpanzees in african sanctuaries. - Plos One 6: e17147. 